\title{
Distance entre les racines d'un polynôme
}

RAIRO - Analyse numérique, tome 13, nº 2 (1979), p. 181-192.

<http://www.numdam.org/item?id=M2AN_1979_13_2_181_0>

(C) AFCET, 1979, tous droits réservés.

L'accès aux archives de la revue «RAIRO - Analyse numérique » implique l'accord avec les conditions générales d'utilisation (http://www.numdam.org/ legal.php). Toute utilisation commerciale ou impression systématique est constitutive d'une infraction pénale. Toute copie ou impression de ce fichier doit contenir la présente mention de copyright.

\section{Numdam}

Article numérisé dans le cadre du programme

Numérisation de documents anciens mathématiques

http://www.numdam.org/ 


\title{
DISTANCE ENTRE LES RACINES D'UN POLYNÔME (*)
}

\author{
par M. Mignotte $\left({ }^{1}\right)$ et M. Payafar $\left({ }^{2}\right)$ \\ Communiqué par P G Ciarlet
}

\begin{abstract}
Résumé - Nous étudions des minorations de la distance entre des racines distinctes d'un polynôme à coefficients entiers Le cas des polynômes cubiques est étudié en détal Nous construisons aussi des polynômes irréductibles sur les entıers ayant deux ractnes « très proches »

Abstract - We study lower bounds of the distance between distinct root of an integer polynomial $A$ detalled study of the case of cubic polynomials is made We also bull irreductible polynomials over the integers uith two "very closed" roots
\end{abstract}

\section{INTRODUCTION}

La séparation des racines d'un polynôme constitue souvent le premier pas des algorithmes de calcul des racines de ce polynôme. Ce type de calcul intervient bien sûr dans certains problèmes d'Analyse Numérique, il apparaît aussi comme étape d'algorithmes de calcul formel et on sait l'importance croissante du Calcul Algébrique Symbolıque. Des discussions plus détaillées sur ce sujet figurent en particulier dans les articles suivants $[1,2,5] \ldots$

Nous considérerons ici surtout des polynômes à coefficients entıers. Ce sont ces polynômes qui interviennent dans les calculs symboliques. En outre, les calculs avec les ordinateurs digitaux sont le plus souvent effectués sur des nombres binaires de longueur bornée et peuvent être ramenés, par un changement d'unité, à des calculs sur les entiers.

Nous étudions des minorations de la distance minimale entre les racines distinctes d'un polynôme. Nous rappelons brièvement les résultats connus et de plus obtenons un certain nombre de minorations nouvelles.

$\left({ }^{*}\right)$ Reçu maı 1978

(1) Centre de Calcul, Université Louıs-Pasteur, Strasbourg (France)

$\left({ }^{2}\right)$ Polytechnıque de Téhéran, avenue Hafez, Téhéran (Iran)

R A I R O Analyse numérıque/Numerical Analys1s, 0399-0516/1979/181/\$ 400

(C) Bordas-Dunod 


\section{NOTATIONS}

Nous considérerons dans toute la suite un polynôme $P$ de degre $d \geqq 2$ a coefficients complexes et de racines $\alpha_{\imath}$,

$$
P(X)=a_{d} X^{d}+\quad+a_{0}=a_{d}\left(X-\alpha_{1}\right) \quad\left(X-\alpha_{d}\right), \quad a_{0} a_{d} \neq 0,
$$

dont les racines sont numerotées de sorte que l'on ait

$$
\left|\alpha_{1}\right| \geqq\left|\alpha_{2}\right| \geqq \quad \geqq\left|\alpha_{d}\right|
$$

La distance mınımale entre deux racınes distınctes de $P$ est la quantıté

$$
\operatorname{sep}(P)=\min _{\alpha \neq \alpha}\left|\alpha_{\imath}-\alpha_{\jmath}\right|,
$$

avec par convention $\operatorname{sep}(P)=\infty$ si $P$ ne possede pas deux racines distinctes La mesure $M(P)$ du polynôme $P$ est définie par la relation

$$
M(P)=\frac{1}{2 \pi} \int_{0}^{2 \pi} \log \left|P\left(e^{\imath \theta}\right)\right| d \theta,
$$

la formule de Jensen montre que

$$
M(P)=\left|a_{d}\right| \prod_{\imath=1}^{d} \max \left\{1,\left|\alpha_{\imath}\right|\right\}
$$

Le discriminant $\Delta$ du polynôme $P$ vaut

$$
\Delta=a_{d}^{2 d-2} \prod_{i<1}\left(\alpha_{\imath}-\alpha_{\jmath}\right)^{2},
$$

s1 les $a_{\imath}$ sont tous entiers alors $\Delta$ est aussi un entier

On posera enfin

$$
\|P\|_{2}=\left(\sum_{i=0}^{d}\left|a_{i}\right|^{2}\right)^{1 / 2}
$$

\section{MAJORATION DE LA MESURE D'UN POLYNÔME}

Le résultat suivant a été démontré récemment par W Lawton [4]

Lemme 1 - Soit $P$ un polynôme donné par la formule (1) Pour tout polynôme $Q$ on pose

$$
\|Q\|=\left(\frac{1}{2 \pi} \int_{0}^{2 \pi}\left|Q\left(e^{\imath \theta}\right) P\left(e^{\imath \theta}\right)\right|^{2} d \theta\right)^{1 / 2}
$$

R A I R O Analyse numerique/Numerical Analysis 
On a alors :

(i) $M(P)^{2}=\inf _{Q, Q(0)=1}\|Q\|$;

(ii) $\inf _{Q, Q(0)=1, \operatorname{deg}(Q)=l}\|Q\|=g_{l+1} / g_{l}$;

où on a posé

$$
g_{l}=\operatorname{det}(\varphi(i-j))_{1 \leqq l, \jmath \leqq l}
$$

et

$$
\varphi(k)=\left\{\begin{array}{c}
a_{d} \bar{a}_{d-k}+a_{d-1} \bar{a}_{d-k-1}+\ldots+a_{k} \bar{a}_{0} \quad \text { si } 0 \leqq k \leqq d, \\
0 \text { si } k>d, \\
\varphi(-k) \quad \text { si } k<0 .
\end{array}\right.
$$

On en déduit le résultat bien connu suivant :

Corollaire 1. - La mesure de P vérifie :

$$
M(P) \leqq\|P\|_{2} .
$$

$>$ On a $M(P) \leqq\left\|Q_{0}\right\|$ pour $Q_{0}(X)=1$. D'après la formule de Parseval $\left\|Q_{0}\right\|=\|P\|_{2} \cdot<$

Ce corollaire peut être raffiné en appliquant la formule (ii) pour $l=1$. On obtient ainsi l'inégalité suivante, qui ne semble pas avoir mise en évidence auparavant.

Corollaire 2. - Si P est un polynôme donné par la formule (1) alors sa mesure vérifie :

$$
M(P)^{2} \leqq\|P\|_{2}^{2}-\left|a_{d} \bar{a}_{d-1}+a_{d-1} \bar{a}_{d-2}+\ldots+a_{1} \bar{a}_{0}\right|^{2} .
$$

\section{MINORATIONS GENERALES DE $\left|\alpha_{2}-\alpha_{\jmath}\right|$}

Nous distinguerons plusieurs cas, en supposant toujours $i<j$. Rappelons que la numérotation choisie pour les racines de $P$ est telle que l'on a $\left|\alpha_{1}\right| \geqq\left|\alpha_{2}\right| \geqq \ldots \geqq\left|\alpha_{d}\right|$.

\section{a) Cas général}

Majorons le discriminant $\Delta$ du polynôme $P$. On a

$$
\begin{gathered}
|\Delta|=\left|a_{d}^{2 d-2}\left(\alpha_{i}-\alpha_{j}\right)^{2} \prod_{\substack{1 \leqq k<k^{\prime} \leqq d \\
k \neq i}}\left(\alpha_{k}-\alpha_{k^{\prime}}\right)^{2} \prod_{\substack{k>1 \\
k \neq j}}\left(\alpha_{k}-\alpha_{i}\right)^{2}\right| \\
\leqq\left|\alpha_{i}-\alpha_{j}\right|^{2}\left|a_{d}\right|^{2 d-2} \prod_{\substack{1 \leqq k<k^{\prime} \leqq d \\
k \neq 1}}\left(2\left|\alpha_{k}\right|\right)^{2} \prod_{\substack{k>1 \\
k \neq j}}\left(2\left|\alpha_{i}\right|\right)^{2}
\end{gathered}
$$

vol. $13, \mathrm{n}^{\circ} 2,1979$ 


$$
\begin{aligned}
=\left|\alpha_{l}-\alpha_{j}\right|^{2}\left|a_{d}\right|^{2 d-2}( & \left.\prod_{1 \leqq k<d}\left(2\left|\alpha_{k}\right|\right)^{2(d-k)}\right)\left(2\left|\alpha_{j}\right|\right)^{-2} \\
=\left|\alpha_{l}-\alpha_{j}\right|^{2} & \left|a_{d}\right|^{2 d-2} 2^{d(d-1)-2} \\
& \times\left|\alpha_{1}\right|^{2(d-1)}\left|\alpha_{2}\right|^{2(d-2)} \ldots\left|\alpha_{d-1}\right|^{2}\left|\alpha_{J}\right|^{-2}
\end{aligned}
$$

Par conséquent,

$$
\left|\alpha_{\iota}-\alpha_{J}\right| \geqq|\Delta|^{1 / 2} 2^{1-d(d-1) / 2}\left|\alpha_{J}\right|\left|a_{d}\right|^{1-d}\left|\alpha_{1}\right|^{1-d}\left|\alpha_{2}\right|^{2-d} \ldots\left|\alpha_{d-1}\right|^{-1} \text {, }
$$

et en particulier

$$
\left|\alpha_{\imath}-\alpha_{J}\right| \geqq|\Delta|^{1 / 2} 2^{1-d(d-1) / 2} M(P)^{1-d}
$$

Plus généralement, soit $\Omega \subset\{(i, j) ; 1 \leqq i<j \leqq d\}$ et soit $\Omega^{\prime}=\{J ;(i, J) \in \Omega\}$ alors l'inégalité (4) peut être remplacée par

$$
\prod_{(l, j) \in \Omega}\left|\alpha_{l}-\alpha_{J}\right| \geqq|\Delta|^{1 / 2} 2^{k-d(d-1) / 2} \prod_{j \in \Omega}\left|\alpha_{j}\right| \cdot\left|a_{d}\right|^{1-d}\left|\alpha_{1}\right|^{1-d} \ldots\left|\alpha_{d-1}\right|^{-1},
$$

où $k=\operatorname{Card}(\Omega)$.

Les majorations précédentes de $M(P)$ permettent d'obtenir des minorations de $\operatorname{sep}(P)$ qui s'expriment facilement en fonction des coefficients de $P$. C'est ainsi que des inégalités (2) et (5) on déduit la minoration

$$
\operatorname{sep}(P) \geqq 2^{2-d(d-1) / 2}|\Delta|^{1 / 2}\|P\|_{2}^{1-d}
$$

Remarque 1. - Dans les formules précédentes la dépendance en fonction de $d$ est assez médiocre puisque de la forme $c^{-d^{2}}$. On peut remplacer ce terme dans la formule (5) $\operatorname{par} d^{-c d}$ en procédant ainsi. Remarquons d'abord que le discriminant de $P$ est aussı donné par la formule

$$
\Delta= \pm a_{d}^{2 d-2}\left(\operatorname{det}\left(\alpha_{k}^{l}\right)_{0<\mathrm{k} \leqq \mathrm{d}, 0 \leqq l<d}\right)^{2} .
$$

Par une manipulation élémentaire du déterminant $D$ qui figure au membre de droite et l'inégalité de Hadamard, on obtient facilement

$$
\begin{aligned}
|D| \leqq\left(\sum_{k=1}^{d-1}\left|\alpha_{\imath}^{h}-\alpha_{j}^{h}\right|^{2}\right)^{1 / 2} \prod_{k \neq 1} & \left(1+\left|\alpha_{k}\right|^{2}+\ldots+\left|\alpha_{k}\right|^{2 d-2}\right)^{1 / 2} \\
& <\left|\alpha_{\imath}-\alpha_{J}\right| d^{(d+2) / 2} \max \left\{1,\left|\alpha_{\imath}\right|\right\}^{-1}\left(M(P) / a_{d}\right)^{d-1} .
\end{aligned}
$$

D'où la minoration

$$
\left|\alpha_{\imath}-\alpha_{J}\right|>|\Delta|^{1 / 2} \max \left\{1,\left|\alpha_{\imath}\right|\right\} d^{-(d+2) / 2} M(P)^{1-d},
$$


qui implique

$$
\operatorname{sep}(P)>d^{-(d+2) / 2}|\Delta|^{1 / 2}\|P\|_{2}^{1-d},
$$

inégalıté quı peut être amélıorée par le facteur multiplicatıf $\sqrt{3}$ ([3], Th. 1).

Quol qu'il en solt nous nous intéressons ici surtout à la dépendance en fonction de $M(P)$ et nous ne chercherons pas des formules raffinées en fonction de $d$.

Notons encore que dans le cas partıculier (important) des polynômes à coefficients entiers sans facteurs multiples on a $|\Delta| \geqq 1$, ce qui simplifie les inégalités ci-dessus.

L'inégalité (6) va nous permettre d'améliorer l'inégalité (5) dans certains cas que nous examinons maintenant.

b) $\alpha_{l}$ et $\alpha_{j}$ non réels, $\alpha_{J} \neq \bar{\alpha}_{l}, P$ à coefficients réels.

Appliquons (6) avec $\Omega=\left\{(i, j),\left(i^{\prime}, j^{\prime}\right)\right\}$ où $\alpha_{i^{\prime}}=\bar{\alpha}_{\imath}, \alpha=\bar{\alpha}_{j}$. Il vient

$$
\left|\alpha_{\imath}-\alpha_{J}\right| \geqq|\Delta|^{1 / 4} 2^{1-d(d-1) / 4}\left|\alpha_{J}\right|\left(\left|a_{d}\right|^{1-d}\left|\alpha_{1}\right|^{1-d} \ldots\left|\alpha_{d-1}\right|^{-1}\right)^{1 / 2},
$$

donc aussi

$$
\left|\alpha_{\imath}-\alpha_{\jmath}\right| \geqq|\Delta|^{1 / 4} 2^{1-d(d-1) / 4} M(P)^{(1-d) / 2} .
$$

Les inégalités (5) et (9) figurent essentiellement dans l'article [3] de Güting. Par contre le cas suivant semble nouveau.

c) $\alpha_{2}$ réel, $\alpha$, non réel, $P$ à coefficients réels.

Si $\alpha_{j}=\bar{\alpha}_{J}$ on prend $\Omega=\left\{(i, j),\left(i, j^{\prime}\right),\left(j, j^{\prime}\right)\right\}$ et on applique la formule (6), en remarquant que l'on a

d'où l'ınégalité

$$
\left|\alpha_{\jmath}-\bar{\alpha}_{\jmath}\right| \leqq 2\left|\alpha_{\imath}-\alpha_{\jmath}\right|
$$

$$
\left|\alpha_{\imath}-\alpha_{J}\right| \geqq\left(|\Delta|^{1 / 2} 2^{2-d(d-1) / 2}\left|\alpha_{J}\right|^{3}\left|a_{d}\right|^{1-d}\left|\alpha_{1}\right|^{1-d} \ldots\left|\alpha_{d-1}\right|^{-1}\right)^{1 / 3},
$$

qui implique

$$
\left|\alpha_{\imath}-\alpha_{\jmath}\right| \geqq\left(|\Delta|^{1 / 2} 2^{2-d(d-1) / 2} M(P)^{1-d}\right)^{1 / 3} .
$$

Remarquons que si $\alpha_{J}$ est réel et $\alpha_{\imath}$ non réel (et si les coefficients de $P$ sont réels) la minoration (10) a encore lieu.

En résumé, nous obtenons le théorème suivant.

Theoreme 1. - Soit $P$ un polynôme de degré d à coefficients complexes, de discriminant $\Delta$, de racines distinctes $\alpha_{1}, \ldots, \alpha_{d}$ avec $\left|\alpha_{1}\right| \geqq\left|\alpha_{2}\right| \geqq \ldots \geqq\left|\alpha_{d}\right|$,

$$
P(X)=a_{d} X^{d}+\ldots+a_{0}, \quad d \geqq 2 .
$$

vol $13, n^{\circ} 2,1979$ 
Alors on a l'inégalité

$$
\left|\alpha_{\imath}-\alpha_{J}\right| \geqq|\Delta|^{1 / 2} 2^{1-d(d-1) / 2}\left|\alpha_{\jmath}\right| \cdot\left|a_{d}\right|^{1-d}\left|\alpha_{1}\right|^{1-d}\left|\alpha_{2}\right|^{2-d} \ldots\left|\alpha_{d-1}\right|^{-1} .
$$

De plus, lorsque $P$ est à coefficients réels, si $\alpha_{\imath}$ et $\alpha_{\text {J }}$ sont des racines imaginaires de $P$ non conjuguées on a

$$
\left|\alpha_{2}-\alpha_{J}\right| \geqq|\Delta|^{1 / 4} 2^{1-d(d-1) / 4}\left|\alpha_{j}\right|\left(\left|a_{d}\right|^{1-d}\left|\alpha_{1}\right|^{1-d}\left|\alpha_{2}\right|^{2-d} \ldots\left|\alpha_{d-1}\right|^{-1}\right)^{1 / 2}
$$

et si un des nombres $\alpha_{1}, \alpha_{j}$ et un seul est réel alors :

$$
\left|\alpha_{\imath}-\alpha_{J}\right| \geqq\left(|\Delta|^{1 / 2} 2^{2-d(d-1) / 2}\left|\alpha_{j}\right|^{3}\left|a_{d}\right|^{1-d}\left|\alpha_{1}\right|^{1-d}\left|\alpha_{2}\right|^{2-d} \ldots\left|\alpha_{d-1}\right|^{-1}\right)^{1 / 3} \text {. }
$$

\section{UTILISATION DE PROPRIÉTÉS GALOISIENNES}

Supposons le polynôme $P$ à coefficients entiers et ırréductıble sur le corps $\mathbf{Q}$ des rationnels. Soit $D$ le degré sur $\mathbf{Q}$ du corps $\mathbf{Q}\left(\alpha_{l}, \alpha_{\jmath}\right)$, alors $d \leqq D \leqq d(d-1)$. Considérons le nombre

$$
q=a_{d}^{2 \delta} \prod_{\sigma} \sigma\left(\alpha_{\imath}-\alpha_{\jmath}\right) \quad \text { avec } \delta=D / d,
$$

où $\sigma$ parcourt l'ensemble $E$ des plongements du corps $\mathbf{Q}\left(\alpha_{\imath}, \alpha_{\jmath}\right)$ dans $\mathbf{C}$. Le nombre $q$ est un entier rationnel non nul, donc

$$
1 \leqq|q| \leqq\left|\alpha_{\imath}-\alpha_{J}\right| \cdot\left|a_{d}\right|^{2 \delta} \prod_{\sigma \neq \mathrm{Id}}\left(\left|\sigma\left(\alpha_{\imath}\right)\right|+\left|\sigma\left(\alpha_{j}\right)\right|\right) .
$$

En utilisant le fait que pour chaque $\alpha_{k}$ la famille $\left(\sigma\left(\alpha_{k}\right)\right)_{\sigma \in t}$ comporte chaque élément $\alpha_{k}$ répété exactement $\delta$ fois, on obtient :

où

$$
1 \leqq\left|\alpha_{1}-\alpha_{J}\right| \cdot\left|a_{d}\right|^{2 \delta} 2^{D-1}\left|\alpha_{1}^{2 \delta} \alpha_{2}^{2 \delta} \ldots \alpha_{\delta-1}^{2 \delta} \alpha_{\delta}^{\varepsilon \delta-1}\right|
$$

$$
\delta^{\prime}=[(\delta+1) / 2] \quad \text { et } \quad \varepsilon=1+2 \delta^{\prime}-\delta \quad(=1 \text { si } \delta \text { est pair et } 2 \text { sinon }) .
$$

D'où la mınoration

$$
\left|\alpha_{\imath}-\alpha_{j}\right| \geqq 2^{1-D}\left|a_{d}\right|^{-2 \delta}\left|\alpha_{1}^{2 \delta} \alpha_{2}^{2 \delta} \ldots \alpha_{\delta^{\prime}-1}^{2 \delta} \alpha_{\delta}^{\varepsilon \delta-1}\right|^{-1}
$$

qui implique

$$
\left|\alpha_{\imath}-\alpha_{\jmath}\right| \geqq 2^{1-D} M(P)^{-2 \delta} .
$$

D'où le résultat suivant :

THÉORÈME 2. - Soit $P$ un polynôme à coefficients entiers trréductible sur $\mathbf{Q}$, de degré d et de mesure $M(P)$. Soient $\alpha_{\imath}$ et $\alpha_{J}$ deux racines distinctes de $P$ et soit $\delta$ le degré du corps $\mathbf{Q}\left(\alpha_{\imath}, \alpha_{\mathrm{j}}\right)$ sur le corps $\mathbf{Q}\left(\alpha_{\imath}\right)$ alors on a

$$
\left|\alpha_{\imath}-\alpha_{\jmath}\right| \geqq 2^{1-d \delta} M(P)^{-2 \delta} \text {. }
$$


Remarquons que l'négalıte (5) jointe à la mınoratıon $|\Delta| \geqq 1$ (justıfiee par le fait que $P$ est $1 \mathrm{c}$ un polynôme irréductıble à coefficients entıers) conduit à la relation

$$
\left|\alpha_{\imath}-\alpha_{\jmath}\right| \geqq 2^{1-d(d-1) / 2} \mathrm{M}(\mathrm{P})^{1-d},
$$

par conséquent la formule (12) est plus précıse que cette dernière inégalité lorsque $D$ vérıfie $D<d(d-1) / 2$, ce qui ne peut se produrre que pour $d \geqq 4$

\section{APPLICATION DES RÉSULTATS PRECEDENTS AUX POLYNÔMES DE DEGRE 3}

Nous avons choisı pour 1llustrer les résultats précédents le premıer exemple non trivial, celuı des polynômes de degre 3 à coefficients entiers sans racıne multiple

1 Solt donc $P=a_{3} X^{3}+a_{2} X^{2}+a_{1} X+a_{0}$ un polynôme de degré 3 a coefficients entiets de racines $\alpha_{1}, \alpha_{2}, \alpha_{3}$ distinctes avec $\left|\alpha_{1}\right| \geqq\left|\alpha_{2}\right| \geqq\left|\alpha_{3}\right|$

Supposons d'abord que $P$ ne possede qu'une racine réelle Appelons $\alpha$ la racıne réelle et $\beta, \bar{\beta}$ les racınes complexes conjuguees de $P$ Les inégalités (10) et (4) impliquent respectivement

$$
|\alpha-\beta| \geqq(2 M(P))^{-2 / 3}
$$

et

$$
|\beta-\bar{\beta}| \geqq\left|2 a_{3} \alpha_{1}\right|^{2} \geqq(2 M(P))^{-2}
$$

Passons maintenant au cas géneral D'apres (4), nous avons alors .

$$
\left|\alpha_{2}-\alpha_{J}\right| \geqq 2^{-2} a_{3}^{-2}\left|\alpha_{1}\right|^{-2} \quad \text { pour } \quad \imath \neq J
$$

Il est à noter que les inégalıtés (14) et (15) ne peuvent être améliorées qu'à une constante multıplicative près En effet, la suite de polynômes

$$
P_{n}(X)=\left(q_{n} X-p_{n}\right)\left(X^{2}-2\right),
$$

où $p_{n} / q_{n}$ est la $n$-1eme convergente du developpement en fraction contınue de $\sqrt{2}$, verifie suivant la parité de $n$

$$
\left|\alpha_{1}-\alpha_{1}\right| \leqq 3 q_{n}^{-2} \alpha_{1}^{-2} \quad \text { pour } \quad l=2 \text { ou } 3
$$

(puisque $\left|p_{n} / q_{n}-\sqrt{2}\right|<q_{n}{ }^{2}$ et que l'on a $p_{n} / q_{n}<\sqrt{2}$ ou $p_{n} / q_{n}>\sqrt{2}$ suivant la parıté de $n$ )

Mais les polynômes ainsı construits sont réductıbles et le problème de l'optımalité de la relation (15) pour des polynômes irréductıbles reste ouvert, aınsı que celuı de l'optımalité des relations (13) et (14)

vol $13 \mathrm{n}^{\circ} 21979$ 
2 Considérons maintenant le cas particulier important ou $P$ est un polynôme unitaire, autrement dit supposons $a_{3}=1$ On a alors d'après (4)

$$
\left|\alpha_{1}-\alpha_{2}\right| \geqq 2^{-2}\left|\alpha_{1}\right|^{-2}
$$

Supposons $\left|\alpha_{1}-\alpha_{2}\right| \leqq 1 / 5$, alors

$$
\left.\left|\alpha_{1}\right|^{2}<\left|\alpha_{1}\right|\left(\left|\alpha_{2}\right|+\frac{1}{4}\right) \leqq \frac{5}{4}\left|\alpha_{1} \alpha_{2}\right| \leqq \frac{5}{4} M(P) \quad \text { (puisque }\left|\alpha_{1}\right| \geqq 1\right),
$$

donc

$$
\left|\alpha_{1}-\alpha_{2}\right|>(5 M(P))^{-1}
$$

Inégalité encore vérifiee pour $\left|\alpha_{1}-\alpha_{2}\right|>1 / 5$

3 Un cas encore plus partıculier est celuı des polynômes cubiques unitaires et dont le second coefficient, $a_{2}$, est nul C'est auss1 un cas important en pratique Supposons donc maintenant $a_{3}=1$ et $a_{2}=0$ La somme des racines de $P$ est nulle Il en résulte que $P$ ne peut avoir une seule racine de grand module Plus précisement, la relation

implique

$$
\alpha_{1}+\alpha_{2}+\alpha_{3}=0
$$

$$
\left|\alpha_{1}\right| \leqq 2\left|\alpha_{2}\right|
$$

On en déduit la majoration

$$
\left|\alpha_{1}\right| \leqq \sqrt{2 M(P)}
$$

qui, jointe a (15), fournit l'inegalite

$$
\left|\alpha_{\imath}-\alpha_{J}\right| \geqq(8 M(P))^{-1} \quad \text { pour } \quad \imath \neq J
$$

Résumons les resultats obtenus

Theoreme 3 - Solt $P(X)=a_{3} X^{3}+a_{2} X^{2}+a_{1} X+a_{0}$ un polynôme cubrque à coefficients entiers de racines distinctes $\alpha_{1}, \alpha_{2}, \alpha_{3}\left(\right.$ avec $\left.\left|\alpha_{1}\right| \geqq\left|\alpha_{2}\right| \geqq\left|\alpha_{3}\right|\right)$ Alors on a toujours

$$
\left|\alpha_{l}-\alpha_{J}\right| \geqq\left(2 a_{3}\left|\alpha_{1}\right|\right)^{-2} \quad \text { pour } \quad l \neq J
$$

Sl $\alpha_{\imath}$ est réelle et $\alpha_{J}$ ne l'est pas alors

$$
\left|\alpha_{t}-\alpha_{\jmath}\right| \geqq(2 M(P))^{-2 / 3}
$$

Lorsque P est unttalre $\alpha_{1}$ et $\alpha_{2}$ ver fient

$$
\left|\alpha_{1}-\alpha_{2}\right|>(5 M(P))^{-1}
$$

Enfin sl $a_{3}=1$ et $a_{2}=0$, on $a$

$$
\operatorname{sep}(P) \geqq(8 M(P))^{1}
$$

R A I R O Analyse numerıque/Numerical Analysıs 


\section{7. ÉTUDE ASYMPTOTIQUE}

Nous nous proposons d'étudier pour $d$ fixé le comportement de sep $(P)$ lorsque $\|P\|_{2}$ tend vers l'infini. Nous considèrerons plus précisément la quantité

$$
\rho(P):=\frac{-\log (\operatorname{sep}(P))}{\log \left(\|P\|_{2}\right)},
$$

$P$ parcourant l'ensemble $U_{d}$ des polynômes de degré $d$ à coefficients entiers et sans racine multiple. Pour un tel polynôme, l'inégalité (7) nous apprend que l'on a

Posons

$$
\rho(P) \leqq(d-1)+d^{2}\left(\log \|P\|_{2}\right)^{-1} .
$$

$$
L(d)=\lim _{P \in U_{d}} \sup _{\|P\|_{2} \rightarrow \infty} \rho(P) .
$$

Cette fonction a déjà été étudiée en [5], où elle est définie par une formule légèrement différente. La majoration précédente de $\rho(P)$ montre que l'on a

$$
L(d) \leqq d-1 .
$$

En utilisant des résultats de Wirsing et W. Schmidt, il a été démontré dans ce travail la minoration

$$
L(d) \geqq[(d+1) / 2]
$$

On a donc

$$
\begin{aligned}
& L(2)=1 \\
& L(3)=2 .
\end{aligned}
$$

La première de ces égalités est presque triviale, la seconde résoud un problème posé par Collins et Horowitz en [1]. Pour $d \geqq 4$ la valeur de $L(d)$ ne semble pas connue.

La démonstration de l'inégalité (19) qui figure en [5] est obtenue en construisant des polynômes réductibles ayant un facteur fixe $Q$ de degré $[d / 2]+1$ et un autre facteur dont une racine tend vers une racine donnée de $Q$. Cette remarque conduit à considérer la fonction

$$
L^{*}(d)=\lim _{P \in U_{d}} \sup _{P \text { iréductuble }} \rho(P) \text { quand }\|P\|_{2} \rightarrow \infty .
$$

Du fait de l'importance pratique des polynômes unitaires il est encore naturel de considérer les quantités

$$
L_{0}(d)=\lim _{P \in U_{d}} \sup _{P \text { untare }} \rho(P)
$$

vol. $13, \mathrm{n}^{\circ} 2,1979$ 
et

$$
L_{0}^{*}(d)=\lim _{P \in U_{d}} \sup _{\substack{P \text { unitare } \\ \text { et arreductible }}} \rho(P)
$$

Ces fonctions vérifient trivialement les ınégalités

et

$$
L_{0}^{*}(d) \leqq L^{*}(d) \leqq L(d)
$$

$$
L_{0}^{*}(d) \leqq L_{0}(d) \leqq L(d)
$$

Il est facile de voir que l'on a

$$
L^{*}(2)=1, \quad L_{0}^{*}(2)=L_{0}(2)=0
$$

Pour $d \geqq 3$ ll semble que l'on ne connaît aucune des valeurs $L_{0}^{*}(d), L_{0}(d)$ ou $L^{*}(d)$

Nous allons démontrer des minorations de $L_{0}^{*}(d)$

TheOREME $4-$ Pour $d \geqq 3$ on $a$

$$
L_{0}^{*}(d) \geqq 1 / 2
$$

et

$$
L_{0}^{*}(d) \geqq d^{1 / 3} / 15 \quad \text { pour } \quad d \geqq 24
$$

$>$ La démonstration de (20) est facıle On considère la famılle de polynômes

$$
P_{k}(X)=X^{d}-(4 k+2) X^{2}+2, \quad k \rightarrow \infty,
$$

le polynôme $P_{k}$ a deux racines proches de $\pm(2 k)^{-1 / 2}$ et le critère d'Eisensteın montre qu'il est irréductıble

Le schéma de la démonstration de la minoration (21) est le suivant Soit $n=\left[(d / 3)^{1 / 3}\right]$ et soit $H$ un entier très grand D'après (19) on peut trouver un polynôme $Q$ de degré $n$ à coefficients entıers et qui vérifie $\rho(Q) \geqq n / 2-1 /(2 n)$ et $\|Q\|_{2} \geqq H$ Pour $|x|>1$ et $|y|>1$ on a $\left|x^{-1}-y^{-1}\right|<|x-y|$, on peut donc supposer que $Q$ a deux racines $\alpha$ et $\beta$ avec $|\alpha| \leqq 1$ et $\operatorname{sep}(Q)=|\alpha-\beta|$ (si tel n'est pas le cas remplacer $Q$ par son polynôme reciproque)

Solt $k=\left[\|Q\|_{2}^{1 / n}\right]+1$ alors le polynôme $Q(k X)$ possède les racınes $\alpha^{\prime}=\alpha / k$ et $\beta^{\prime}=\beta / k$ et on $a$

$$
\begin{gathered}
\max \left\{\left|\alpha^{\prime}\right|,\left|\beta^{\prime}\right|\right\} \leqq 2\|Q\|_{2}^{-1 / n}, \\
2\left|\alpha^{\prime}-\beta^{\prime}\right| \leqq\|Q\|_{2}^{-n / 2}
\end{gathered}
$$

Soit $p$ le plus petit nombre premier qui ne divise pas $Q(0)$, on pose

$$
R(X)=p Q(k X), \quad P(X)=X^{d}+R(X),
$$


d'après le critère d'Eisenstein $P$ est irréductible.

Il existe un disque centré au point $\left(\alpha^{\prime}+\beta^{\prime}\right) / 2$ et de rayon majoré par $\left|\alpha^{\prime}-\beta^{\prime}\right|$ qui contient $\alpha^{\prime}$ et $\beta^{\prime}$ dans son intérieur et sur la frontière $\Gamma$ duquel on a

$$
|R(z)| \geqq c_{1}\|R\|_{2}^{-n^{2}},
$$

où $c_{1}$ est une quantité positive qui ne dépend que de $d$. D'autre part, (22) et (23) impliquent

$$
\left|z^{d}\right| \leqq(3 / k)^{d} \quad \text { si } \quad z \in \Gamma \text {. }
$$

Il est facile de démontrer la majoration

$$
2\|R\|_{2} \leqq\left\|Q_{2}\right\|^{5 / 2} \quad \text { pour } H \text { assez grand. }
$$

Des trois inégalités précédentes résulte que l'on a

$$
\left|z^{d}\right|<|R(z)| \quad \text { si } \quad z \in \Gamma \quad \text { (puisque } d \geqq 3 n^{2} \text { ). }
$$

Le théorème de Rouché montre enfin qu'il existe au moins deux racines de $P$ dans le disque de frontière $\Gamma$. On a donc

$$
\operatorname{sep}(P) \leqq 2\left|\alpha^{\prime}-\beta^{\prime}\right| \leqq\|P\|_{2}^{-n / 5} \leqq\|P\|_{2}^{-d^{1 / 3} / 15} .
$$

D'où la seconde assertion. $<$

Remarque. - Nous n'avons fait aucun effort pour raffiner l'inégalité (21) car on peut penser qu'il existe une constante positive $c$ telle que l'on ait

$$
L_{0}^{*}(d) \geqq c d \quad \text { pour } \quad d \geqq 3 .
$$

\section{RÉSULTATS EXPÉRIMENTAUX}

Nous avons étudié le cas particulier des polynômes unitaires de degré 3 dont le second coefficient est nul. Soit :

$$
P=X^{3}-a X+b, \quad b \neq 0,
$$

un tel polynôme, son discriminant vaut

$$
\Delta=4 a^{3}+27 b^{2},
$$

nous le supposons non nul. La distance minimale entre deux racines d'un tel polynôme vérifie :

$$
\operatorname{sep}(P) \geqq|\Delta|^{1 / 2}\left(2\left|\alpha_{1}\right|\right)^{-2} \geqq|\Delta|^{1 / 2}(8 M(P))^{-1},
$$

où comme plus haut $\alpha_{1}$, désigne une racine de $P$ de module maximal.

vol. $13, \mathrm{n}^{\circ} 2,1979$ 
On sait que, dans le cas considéré $1 c 1,|\Delta|$ tend vers l'infinı avec $|b|$, mais on ne sait pas démontrer l'inégahté

$$
\varliminf_{\lim } \frac{\log |\Delta|}{\log |b|}>0
$$

Des calculs, effectués sur UNIVAC 1110 , ont conduit au résultat suivant : pour $a \leqq 23 \cdot 10^{5}$ on a

$$
\varepsilon:=\frac{\log |\Delta|}{\log |b|}>1 / 2 \quad \text { si } \quad \Delta \neq 0
$$

sauf dans les cas qui figurent dans le tableau suıvant:

\begin{tabular}{|c|r|c|c|}
\hline$a$ & \multicolumn{1}{|c|}{$b$} & $\varepsilon$ & $\Delta=27 b^{2}-4 a^{3}$ \\
\hline 17977 & 927735 & 0,481 & 743 \\
\hline 338815 & 75908788 & 0,495 & 7988 \\
\hline 1013692 & 392832257 & 0,275 & -232 \\
\hline 1072283 & 427378081 & 0,449 & -7600 \\
\hline 1306999 & 575123345 & 0,490 & 19680 \\
\hline 2160342 & 1222170726 & 0,482 & 24320 \\
\hline
\end{tabular}

Notons pour conclure que l'on a l'encadrement

$$
\theta \leqq \lim _{P=X^{3}-a X+b} \sup _{b \Delta(P) \neq 0} \rho(P) \leqq 1-\operatorname{Lim} \operatorname{mf} \varepsilon(P) .
$$

\section{BIBLIOGRAPHIE}

1. G. E. Collins and E. Horowitz, The Minimum Root Separation of a Polynomial, Math Comp., vol. 28, ${ }^{\circ} 126,1974$, p. 589-597

2. G E Collins and R. Loos, Polynomial Real Root Isolation by Differentiation, Proceedings of the 1976 A.C.M. Symposium on Symbolic and Algebraic Computation, R D. JENKs, éd., p 15-25.

3 R. Guting, Polynomials with Multiple Zeroes, Mathematika, vol 14, 1967, p. 181196.

4. W. Lawton, Heights of Algebraic Numbers and Szego's Theorem, Proc American Math. Soc, vol. 49, 1975, p. 47-50

5 M. Mignotte, Sur la complexité de certains algorithmes ou intervient la séparatıon des racınes d'un polynôme, R A.I.R.O. Informatıque théorıque, vol 10, 1976, p 51-55. 